\title{
Evaluation of in vitro neuronal networks for the study of spontaneous activity
}

\author{
Diletta POZZI ${ }^{\mathrm{a}, \mathrm{b}^{*}}$ \\ ${ }^{a}$ Neurobiology Sector, International School for Advanced Studies (SISSA), via Bonomea 265, 34136 Trieste, Italy. \\ ${ }^{b}$ Department of Otolaryngology, Head and Neck Surgery, Stanford University School of Medicine, 300 Pasteur Drive, 94305 Stanford, California. \\ *Correspondence: dpozzi@stanford.edu \\ https://doi.org/10.37175/stemedicine.v1i2.35
}

\begin{abstract}
In the absence of external stimuli, the nervous system exhibits a spontaneous electrical activity whose functions are not fully understood, and that represents the background noise of brain operations. Spontaneous activity has been proven to arise not only in vivo, but in in vitro neuronal networks as well, following some stereotypical patterns that reproduce the time course of development of the mammalian nervous system. This review provides an overview of in vitro models for the study of spontaneous network activity, discussing their ability to reproduce in vivo - like dynamics and the main findings obtained with each particular model. While explanted brain slices are able to reproduce the neuronal oscillations typically observed in anaesthetized animals, dissociated cultures allow the use of patient-derived neurons and limit the number of animals used for sample preparation. Moreover, dissociated neurons can be cultured on three-dimensional (3D) substrates that mimic the extra-cellular matrix of the brain. Depending on the material used, 3D substrates are able to increase neuronal connectivity and synaptic strength.
\end{abstract}

Keywords: Cortical oscillations · Up states · Calcium transients · Neuronal cultures · Brain slices

\section{Introduction}

Spontaneous neural oscillations have been observed by researchers as early as the 1930 s, when the electroencephalography (EEG) machine was invented (1). Specifically, slow oscillations in the frequency of delta waves $(0.4-5 \mathrm{~Hz})$ have first been observed in the cortex of sleeping patients by W Gray Walter (2). Subsequently, slow oscillations in the same frequency range were recorded in anesthetized mammals as well (3). Nevertheless, their function remained one of the most mysterious questions in neuroscience.

A first characterization of neuronal spontaneous oscillations was provided by Steriade et al. in 1993. Measuring the electrical activity of cortical and thalamic neurons in anesthetized cats, they observed oscillations composed of a slow depolarizing phase $(0.8-1.5 \mathrm{~s})$ followed by a long-lasting hyperpolarization. These oscillations had an even lower frequency than the one of delta waves, i.e. less than $1 \mathrm{~Hz}$. Therefore, they were referred to as slow oscillations (4). The duration of the depolarizing phase was reduced by administration of the

Received: Jan 25, 2020; Accepted: Mar 4, 2020.

(c) The Author(s). 2019 This is an Open Access article distributed under the terms of the Creative Commons License (http://creativecommons.org/licenses/by/4.0/) which permits unrestricted use, distribution, and reproduction in any medium or format, provided the original work is properly cited.
N-methyl-D-aspartate (NMDA) blocker ketamine.

In the hippocampus of anesthetized rats, instead, neuronal oscillations have been observed mainly in the higher, theta frequency range $(4-8 \mathrm{~Hz})$. Hippocampal theta waves are generated by the entorhinal input and by CA3 collaterals, through the activation of voltagedependent $\mathrm{Ca}^{2+}$ currents in pyramidal cells. GABAergic and cholinergic neurons contribute as well to the generation of theta waves by feed-forward disinhibition of CA1 pyramidal cells by the interneurons, and cholinergic activation of CA3 (5).

Subsequent studies revealed the mechanisms of neuronal oscillations at the cellular level. In particular, some in vivo intracellular recordings of neostriatal spiny neurons showed fluctuations between a depolarized and an hyperpolarized membrane potential that were referred to as Up and Down states, respectively (6). Subsequently, Constantinople and Bruno demonstrated that this two-state behavior observed in anesthetized rats was eliminated during wakefulness. In addition, they showed that the dynamics of the awake state and under anesthesia were unaltered even after elimination of afferent thalamic input, suggesting an internal cortical neuromodulation (7). In fact, it was subsequently proven that - in both humans and rodents - the large majority of excitatory synapses onto cortical excitatory neurons come from other cortical excitatory neurons, giving the cortex a vast recurrent connectivity (8). 
Synchronized Up and Down states are the cellular equivalent of the slow waves observed - at the network level - from human EEG data (9) to anesthetized animals, and even in in vitro brain slices isolated from the rest of the brain (10) (Figure 1). Since they arise in such different systems, Up and Down states have been proposed to be a default activity pattern resulting from the basic architecture of the network. Nevertheless, they may still have a functional role. From experimental observations of their pathways, Up and Down states are supposed to transfer information across brain regions and to consolidate memories. A current hypothesis is that cortical Up states travel through the entorhinal and visual cortex to the hippocampus phasing the hippocampal rhythm, which in turn transfers its activity pattern - acquired during learning - back to the cortical networks for memory consolidation $(5,11)$. Moreover, the hippocampal theta rhythm is believed to be critical for temporal coding/decoding of active neuronal ensembles and the modification of synaptic weights. Down states, as periods of low synaptic inputs, are thought to be necessary for metabolic restoration (see for review 12).

Spontaneous oscillations have been observed also in developing neuronal networks. An example is represented by synchronous plateau assemblies (SPAs) and giant depolarizing potentials (GDPs) in the immature hippocampus. Driven by synchronized GABAergic neurons, GDPs are thought to shape synaptic currents in the immature network $(13,14)$.

In the regulation of this rhythmic activity, a key role is played by inhibitory interneurons. In fact, interneurons were proven to synchronize the spiking of hippocampal pyramidal cells, thus maintaining homeostatic levels of activity in the brain (15). Moreover, spike-triggered local field potentials measured in humans and monkeys were proven to be composed by inhibitory neurons for the largest part, suggesting that the onset of Up states is more driven by a decrease in inhibition rather than an increase in excitation (16).

Not only Up and Down states, but also spontaneous calcium signals were frequently observed in neuronal networks. In fact, intracellular calcium ions increase as a consequence of action potentials and membrane depolarization, entering the cytosol through voltagegated calcium channels. Spontaneous calcium transients can be considered as indicators of Up and Down states since they follow parallel dynamics, despite being slower (17) (see Figure 1 for a representation). Calcium imaging techniques have become a diffused method for studying network dynamics, since they allow the simultaneous recording of a large number of neurons.

Many of these findings were obtained in vitro. In this review, I discuss the commonly used methods for preparing neuronal samples, the patterns of spontaneous activity that typically arise from each one of these networks and their similarity with in vivo neuronal oscillations. Network disorders are at the basis of several pathologies starting from epilepsy, where an increased synchronization of excitatory neurons is causing seizures. Altered network dynamics are also reported in sleep disorders such as narcolepsy, where a specific neuronal population (hypocretin neurons) is reduced in number, and the EEG rhythms normally observed during sleep are modified (18). Moreover, an impaired connectivity of resting-state networks was observed in schizophrenia by functional magnetic resonance imaging (19). In vitro experimental models can be a powerful tool for a deeper understanding of network disorders, and for drug screening of new neuroactive compounds.

\section{2. "Traditional" dissociated cultures - Methods, applications and spontaneous activity}

Neurons preserve the ability to generate spontaneous electrical pulses even when disrupted from their original connectivity and cultured in vitro. In fact, primary dissociated cultures, obtained from embryonic or postnatal
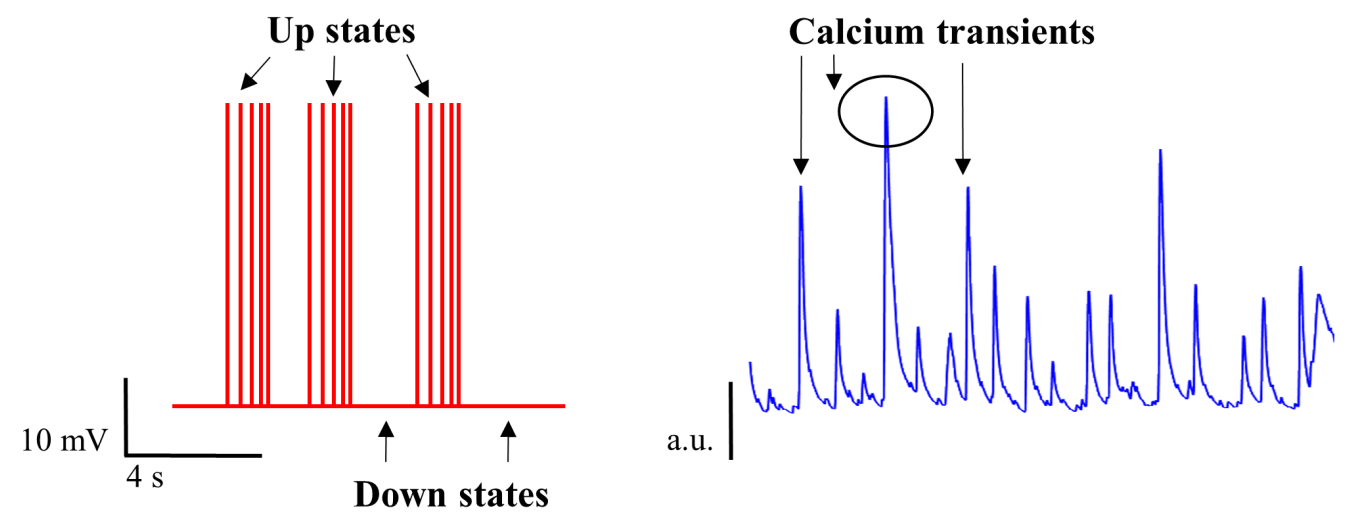

Figure 1. Schematic representation of spontaneous Up and Down states and calcium transients. The red trace on the left represents an example of a typical intracellular recording from a neuron in spontaneous conditions. Up and Down states are visible as rhythmic oscillations between membrane depolarization and hyperpolarization. The blue trace on the right depicts some typical calcium transients recorded from a single neuron in spontaneous condition. The time scale is the same for both traces. Note the increased duration of a single calcium transient comparing to a single Up state event. 
rodent brain regions such as the hippocampus or the cortex, represent a well-established in vitro model for studying neuronal networks, both on a short (network formation) and a long term (differentiation, maturation) scale (20,21). When cells are enzymatically dissociated, plated on suitable substrates and cultured in appropriate media, they grow processes and form ex-novo a functional network. Dissociated cell cultures are traditionally plated on glass-made flat surfaces, allowing a fine space resolution for morphological investigation of small cellular structures, like neurites and growth cones. Neurons attach to surfaces through their anionic phospholipids (e.g. phosphatidylserine) (22), therefore the surface needs to be positively charged. Previous coating with polyaminoacids such as polylysine and polyornithine have since long been used for this purpose, together with the addition of components mimicking the extracellular matrix in situ (i.e. Matrigel $\left.{ }^{\circledR}(23)\right)$. The morphology and development of dissociated neurons as well as their network activity are strongly influenced by the culturing conditions and, in particular, the culturing medium used (24). Different chemical compositions have been tested and are commercially available for neuronal cell cultures, all based on a physiological solution added with a particular supplier of nutrients and growth factors. Fetal bovine serum, for example, provides neurons with sufficient factors for short-term cultures. As any animal-derived product, however, it is prone to batch-to-batch variability (25) and, most of all, it results in a low differentiation of neuronal types (26). For this reason, serum-free media were tested with different additives. A very commonly used one is B27 Supplement ${ }^{\circledR}$, aimed at obtaining almost pure neuronal cultures (27). Another option is to use glial cells, specifically astrocytes, as the intrinsic source of growth factors in the neuronal culture. For this purpose, it is possible to plate neurons on a glial feeder layer (28) or, in order to avoid the spatial interference of astrocytes while maintaining the soluble factors released by them, to grow neurons in an astrocyte-conditioned medium (ACM). ACM is supposed to contain lipids, thrombospondins and Brain Derived Neurotrophic Factor (BDNF), that are essential components for neuronal growth and survival (29). In fact, it was demonstrated to increase the life span of dissociated cultures up to several months $(24,30)$.

Once established the optimal plating and growing conditions for the specific experimental purposes, it is possible to explore the properties of dissociated networks with a fine, single cell resolution. A common application is to analyze different neuronal populations optically labeled. A comparison of spike parameters between the GABAergic and glutamatergic neurons has been possible, in fact, because of the labelling of the former with a green fluorescent protein $(31,32)$.

Network (i.e. synchronized) activity in dissociated neurons emerges at 3 - 7 days in-vitro (DIV) and matures over the following several weeks in culture, as shown by electrophysiological and imaging measurements $(33,34)$. Synchronized neuronal activity has been proven to be mediated by the NMDA receptor, by serotonin and acetylcholine (35). Network activity is also strongly dependent on the initial plating density of neurons: neurons plated at high density present higher rates of less synchronized activity comparing to sparse networks (28). In fact, there is an inverse relationship between the density of the network and its synaptic strength, such as sparse neurons establish less but stronger synaptic connections, as shown also by the longer duration of their bursts. Interestingly, dense neuronal cultures present a peak in synchronicity at DIV15-16, followed by a decrease (36) that resembles the one observed in the intact cortex during development (37).

Recent studies showed some differences in spontaneous, single-unit spike activity between dissociated neurons and awake animals: in vitro, neurons were more likely to fire in synchronized bursts; while in vivo the firing events were mainly composed by single spikes. Moreover, a greater variability was observed in vitro when quantifying the frequency of firing (38). However, as previously mentioned, the variability in spontaneous activity of dissociated neurons can be minimized by adopting a consistent culturing method and, most of all, the same plating density among different preparations.

Dissociated neurons served as a model for studying the progression of neuronal disorders. In a recent study (39), neurons dissociated from a mouse model of Fragile $\mathrm{X}$ syndrome (Fmr1 KO) were recorded by means of microelectrode arrays (MEAs) from 7 to 35 DIV, and exhibited an abnormally increased synchronization of spontaneous firing events at mature stages. Moreover, they showed oscillations in the beta frequency range, which were not observed in controls. These results are in line with previous studies showing network hyperactivity and neuronal hyperconnectivity in the intact cortex of Fmr1 KO mice $(40,41)$.

Finally, dissociated cultures represent the only method for growing human-induced pluripotent stem cells (hiPSCs). These precursors can be differentiated into neurons, allowing the investigation of their molecular and electrophysiological properties $(42,43)$. Most of all, they can be derived from patients for the purpose of specific drug development or cell therapies. Using appropriate differentiation methods, hiPSCs can generate threedimensional (3D) brain organoids composed by neurons and glial cell types (44).

\section{3D neuronal cultures - methods, applications and spontaneous activity}

Traditional, 2D neuronal cultures have been replaced in many studies by more recent $3 \mathrm{D}$ architectures for several reasons. First, neurons cultured on glass coverslips are prone to artifacts due to the flat condition in which they grow. Glass coverslips are in fact distant from the physiological extra-cellular environment of the brain, which is $3 \mathrm{D}$ and soft (less than $1 \mathrm{kPa}$ in Young modulus, while the stiffness of the glass is in the order of GPa). Neurites forced to grow in a planar region present indeed an abnormal excitability (45). Moreover, using plastic materials instead of glass allows for directing the 
differentiation of stem cells towards bone, muscle and neuronal cell types by progressively decreasing the stiffness of the substrate (46). Therefore, 3D substrates with appropriate stiffness were developed for neuronal cultures, and called neuroscaffolds.

An interesting example is represented by the carbonbased neuroscaffolds, engineered for interfacing the cell membrane structures. Carbon-based materials have since long raised the interest of neuroscientists because of the electrical conductivity of the material, that might find its most obvious application in restoring/improving neuronal connectivity $(47,48)$. These materials can be assembled in the form of wrapped graphite sheets, and therefore called carbon nanotubes (CNTs), or in the form of graphene foams. Both cases were proven to be biocompatible and conductive scaffolds not only for culturing primary neurons, but also for neural stem cell differentiation $(49,50)$.

Interestingly, primary hippocampal neurons plated on 3D graphene showed a more frequent and synchronized spontaneous activity comparing to both 2D graphene and traditional 2D glass substrates (51). These results suggest a specific role of dimensionality in network dynamics that is independent from the material where neurons grow. However, a recent study demonstrated that a single layer of graphene modifies the ionic concentration at the interface with neurons so that they will exhibit stronger potassium currents and will shift from adapting to tonically firing. This effect was not observed when neurons were plated on different conductive materials (52). Taken together, these results suggest that both the $3 \mathrm{D}$ structure and the electrical properties of the substrate can increase neuronal firing and connectivity. In fact, adding CNTs as a coating to a $3 \mathrm{D}$ substrate was proven to boost the synaptic activity of dissociated neurons, as shown by calcium imaging experiments (53). For these reasons, carbon-based materials have been tested in in vivo implants for restoring nerve mechanical or chemical injury $(54,55)$ as well as for deep brain stimulation and monitoring $(56,57)$.

Polydimethylsiloxane (PDMS) and other plastic materials represent another interesting category for neuronal studies: while not electrically conductive, these materials have the advantage of being highly ductile and with a Young modulus closer to the one of brain tissues. The fabrication of these types of scaffolds usually requires photolithography techniques combined with micro/nanopatterning (58). Some preliminary treatments - such as plasma cleaning and polylysine/polyornithine coating are necessary to reduce the hydrophobicity of the plastic and ensure neuronal attachment. Plastic micropillars have often been used as substrates for cell cultures in order to decrease the stiffness of the original material: while a flat surface of PDMS has a Young modulus of around $1 \mathrm{MPa}$, PDMS micropillars have a stiffness of 5 - $20 \mathrm{kPa}$ depending on their diameter and height (59). For these reasons, micropillars are useful for determining the mechanical responses and forces exerted by cells (60). Plastic materials can also be molded at the nanoscale: the use of nanopillars with different dimensions and stiffness has been proven to control the adhesion and differentiation of neuronal precursors (61). Moreover, the addition of plastic nanofibers made of poly (lactic-co-glycolic acid) to PDMS micropillars was proven to increase the frequency and amplitude of spontaneous calcium transients in primary hippocampal neurons (62). Due to their biocompatibility, PDMS-based microchannel electrodes have successfully been implanted in rodents for monitoring neural activity over time and for providing a mechanical support for peripheral nerve regeneration (55).

In addition, scaffold-free $3 \mathrm{D}$ cultures can be derived from hiPSCs to generate brain organoids. HiPSCderived organoids have been used for drug screening and modeling of central nervous system diseases (63).

In summary, 3D neuronal cultures recapitulate several aspects of in vivo brain tissue, from synaptic properties of individual neurons to network synchronization. Moreover, comparing to 2D cultures, they offer the unique advantage of recreating organoids with neuronal and glial types interconnected in a cyto-architecture that is able to reproduce the one of the brain.

\section{Explanted brain slices - methods, applications and spontaneous activity}

Slices of the brain or spinal cord can be isolated from the region of interest and directly used for electrophysiological measurements and imaging (17,61). These preparations are referred to as acute slices and, in contrast to dissociated cell cultures, they preserve the native cyto-architecture of the tissue. Acute cortical slices are able to reproduce the most characteristic feature of the cerebral cortex, i.e. slow oscillations between Up and Down states, as shown by the work of Sanchez-Vives starting from the 2000s $(65,66)$. In this work, intracellular recordings from ferret visual and prefrontal cortical slices were performed using microelectrode arrays. While no spontaneous activity was recorded in the "traditional" slice bathing medium $\left(2 \mathrm{mM} \mathrm{Ca}{ }^{2+}, 1 \mathrm{mM} \mathrm{Mg}^{2+}, 2.5 \mathrm{mM}\right.$ $\mathrm{K}^{+}$), some rhythmic oscillations were observed after changing the bath solution to a composition more similar to the brain interstitial fluid (1.0 or $1.2 \mathrm{mM} \mathrm{Ca}^{2+}, 1 \mathrm{mM}$ $\mathrm{Mg}^{2+}$ and $3.5 \mathrm{mM} \mathrm{K}$ ). These oscillations were initiated by pyramidal neurons of layer $\mathrm{V}$, had a periodicity of once every 3.5 seconds and were stable for the life of the slice (12 hours). The cortical slices were prepared from 3 to 8 month-old ferrets and showed a close similarity to those observed in adult, anesthetized cats. In fact, the spontaneous activity of slices was studied as the most likely in vitro equivalent of the activity of the brain in the absence of external stimuli, i.e. during quiescent sleep or under anesthesia. In a more recent study from the same group, variable propagation modes of Up states were observed, suggesting a memoryless generation of slow oscillations (10).

Slices of tissue cultured in vitro for several days are instead referred to as organotypic slice cultures. These 


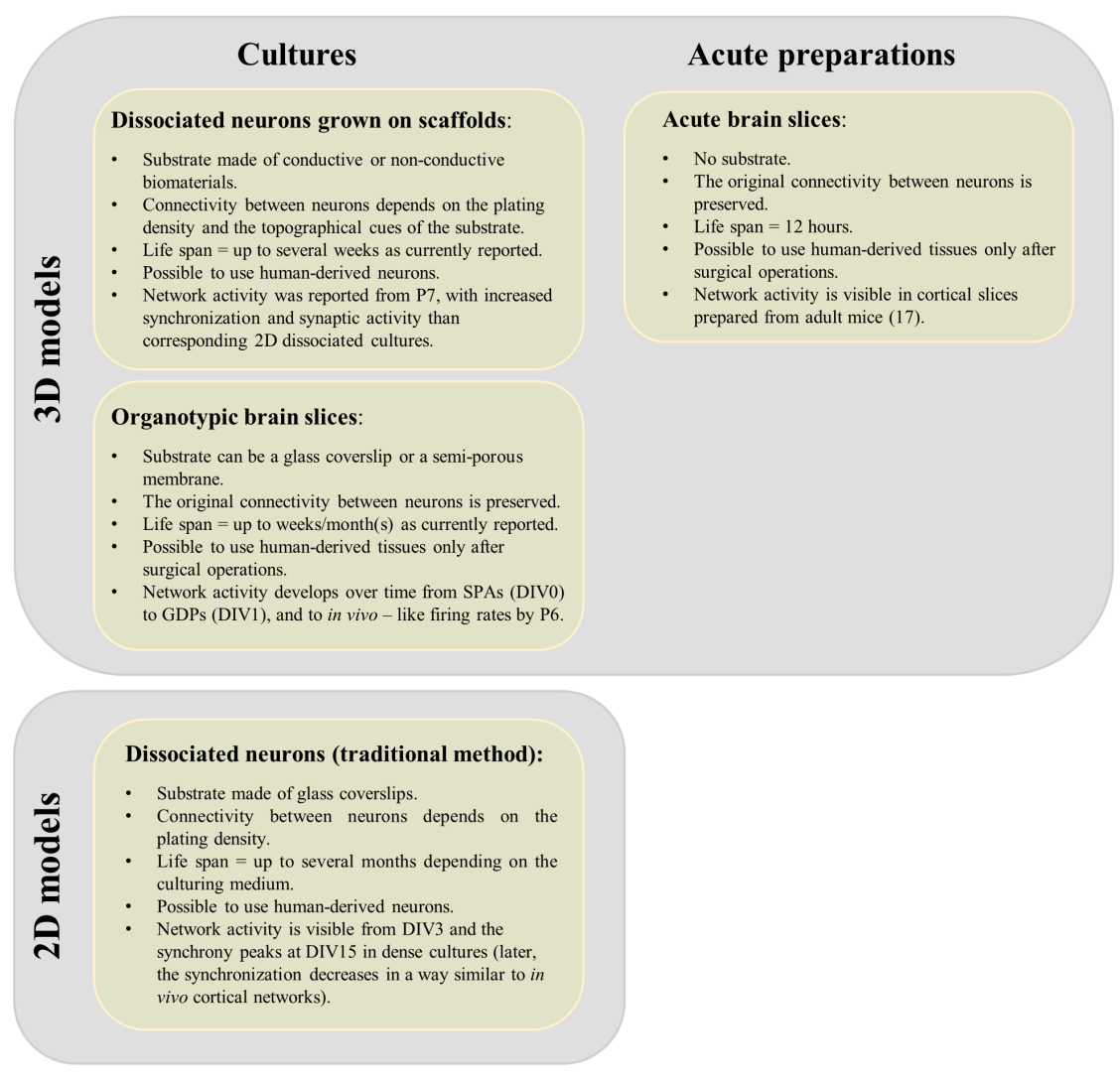

Figure 2. Summary and comparison of in vitro neuronal network models. 3D models include brain slices and dissociated neurons grown on scaffolds. 2D models are represented by dissociated neurons grown on glass (traditional method). The only preparation that does not require culturing is represented by acute brain slices.

preparations were proven to regrow synaptic processes and to restore the functions potentially impaired during the cutting procedure. There are two commonly used methods for preparing organotypic cultures from nervous tissues. In the Gähwiler method (67), slices are cut and attached on glass coverslips by coagulating chicken plasma with thrombin; the coverslips are then inserted into plastic tubes and kept in serum-added culturing medium on a roller drum (ensuring balanced time of oxygenation) at $37^{\circ} \mathrm{C}$. The Stoppini method (68) follows a similar procedure, but the slices are placed on sterile, transparent semiporous membranes in Petri or multiwell dishes, and kept at the interface between air and culturing medium at $37{ }^{\circ} \mathrm{C}$. In this case, it is possible to culture the slices in Neurobasal medium added with B27 supplement, with no addition of animal serum. However, organotypic slices grown in the absence of serum have been reported to develop spontaneous epileptiform activity (69).

The use of organotypic cultures allows to perform chronic multineuron imaging. For example, daily morphological analyses and calcium imaging in hippocampal slices revealed that GABAergic interneurons are involved in the generation of SPAs at DIV0; starting from DIV1, the spontaneous activity of the slice switches to giant depolarizing potentials (70), as in line with previous findings on the development of hippocampal networks (71).

Interestingly, in 2014, Okamoto et al. demonstrated that organotypic hippocampal slices exhibit firing rates and excitatory synaptic activity more similar to those observed in vivo comparing to acute slices (72). In fact, cutting several neurites during the preparation might affect the network activity of acute slices, which do not have time to regrow these processes.

Without the technical issues presented by in vivo measurements (i.e. background noise, the interference of anesthetics and the need of complex surgeries), experiments on slices have been useful to address questions like how inhibitory neurons coordinate synchrony in the network. In a study of 1995 (73), GABAergic neurons were anatomically identified from hippocampal slices and depolarized to produce fast inhibitory postsynaptic potentials: the excitatory, pyramidal neurons synaptically connected to them exhibited some sub-threshold oscillations in the theta frequency range, demonstrating that these spontaneous oscillations in the hippocampus are tuned by the inhibitory activity of GABAergic neurons. Subsequently, a reconstruction of functional connectivity in acute hippocampal slices was performed by Bonifazi et al., combining electrophysiological recordings with calcium imaging: connections in between neurons were assumed 
on the base of the temporal correlations of their activation. Stimulating some specific, highly connected neurons in the network, they observed a higher occurrence of spontaneous GDPs in the network, suggesting the existence of functional hubs that were identified as a subpopulation of GABAergic neurons (64). Results obtained in cortical slices also showed that networks of fast spiking, electrically coupled interneurons coordinate the spontaneous activity (74).

These results are in line with subsequent studies in behaving mice, characterizing the membrane potential dynamics of fast-spiking inhibitory neurons vs excitatory ones (75), and also in human and monkeys during sleep, when the local field potential of the neocortex was measured with MEAs (16). Taken together, these results confirm that brain slices are a suitable in vitro model for investigating network dynamics. They are prepared typically from animal models. However, the use of human brain slices is possible from resected or postmortem tissues $(76,77)$.

\section{Conclusions}

Both dissociated and organotypic cultures exhibit a spontaneous neuronal firing with in vivo-like patterns in their course of development (quantified by days in vitro). Acute and organotypic slices were used for the most remarkable studies on spontaneous neuronal oscillations, given the fact that they preserve the original cyto-architecture of the region of interest. On the other side, 3D substrates made of carbon-based or plastic materials can compensate for the lack of architecture in traditional neuronal cultures and boost the synaptic activity of dissociated neurons, opening the way for neural prostheses and cell culture implants in the central and peripheral nervous system. However, there is no strong evidence that differentiated neurons plated on neuroscaffolds can reproduce the time course of development of intact neuronal networks. HiPSCs, as opposite, are able to reproduce some aspects of human brain development when differentiated into scaffold-free, 3D organoids. HiPSCs offer in fact a powerful model for drug screening and customized therapy. Finally, the use of dissociated neurons and hiPSCs can diminish the number of experimental animals used, making it a more ethical tool for scientific studies and even a pre-clinical test platform for screening of new neuroactive compounds.

For a summary and comparison of the properties of the in vitro models discussed within this review, see Figure 2.

\section{Conflict of interest}

The authors declare that they have no conflict of interest.

\section{References}

1. Berger H. Über das Elektrenkephalogramm des Menschen. Archiv f. Psychiatrie. 1929;87:527-570.

2. Freeman WJ. W.G. Walter: The Living Brain. In: Palm G, Aertsen A, editors. Brain Theory. Springer Berlin Heidelberg; 1986. p. 237-8.

3. Steriade M, Dossi RC, Nuñez A. Network modulation of a slow intrinsic oscillation of cat thalamocortical neurons implicated in sleep delta waves: cortically induced synchronization and brainstem cholinergic suppression. J Neurosci. 1991 Oct;11(10):3200-17.

4. Steriade $M$, Nuñez A, Amzica F. A novel slow (< $1 \mathrm{~Hz}$ ) oscillation of neocortical neurons in vivo: depolarizing and hyperpolarizing components. J Neurosci. 1993 Aug;13(8):3252-65.

5. Sirota $A$, Buzsáki $G$. Interaction between neocortical and hippocampal networks via slow oscillations. Thalamus Relat Syst. 2005 Dec;3(04):245.

6. Wilson CJ, Kawaguchi Y. The origins of two-state spontaneous membrane potential fluctuations of neostriatal spiny neurons. J Neurosci. 1996 Apr 1;16(7):2397-410.

7. Constantinople CM, Bruno RM. Effects and mechanisms of wakefulness on local cortical networks. Neuron. 2011 Mar;69(6):1061-8.

8. Seeman SC, Campagnola L, Davoudian PA, Hoggarth A, Hage TA, Bosma-Moody A, et al. Sparse recurrent excitatory connectivity in the microcircuit of the adult mouse and human cortex. eLife. 2018 Sep 26;7:e37349.

9. Massimini M, Huber R, Ferrarelli F, Hill S, Tononi G. The sleep slow oscillation as a traveling wave. J Neurosci. 2004 Aug 4;24(31):6862-70.

10. Capone C, Rebollo B, Muñoz A, Illa X, Del Giudice P, Sanchez-Vives MV, et al. Slow waves in cortical slices: how spontaneous activity is shaped by laminar structure. Cereb Cortex. 2019 Jan 1;29(1):319-335.

11. Ji D, Wilson MA. Coordinated memory replay in the visual cortex and hippocampus during sleep. Nat Neurosci. 2007 Jan;10(1):100-7.

12. Buzsáki G. Theta Oscillations in the hippocampus. Neuron. 2002 Jan;33(3):325-40.

13. Mohajerani $\mathrm{MH}$, Cherubini $\mathrm{E}$. Role of giant depolarizing potentials in shaping synaptic currents in the developing hippocampus. Crit Rev Neurobiol. 2006;18(1-2):13-23.

14. Ben-Ari Y, Cherubini E, Corradetti R, Gaiarsa JL. Giant synaptic potentials in immature rat CA3 hippocampal neurones. J Physiol. 1989 Sep;416:303-25.

15. Somogyi $P$, Klausberger $T$. Defined types of cortical interneurone structure space and spike timing in the hippocampus. J Physiol. 2005 Jan 1;562(Pt 1):9-26.

16. Teleńczuk B, Dehghani N, Le Van Quyen M, Cash SS, Halgren E, Hatsopoulos NG, et al. Local field potentials primarily reflect inhibitory neuron activity in human and monkey cortex. Sci Rep. 2017 Jan 11;7:40211.

17. Cossart R, Aronov D, Yuste R. Attractor dynamics of network UP states in the neocortex. Nature. 2003 May 15;423(6937):283-8.

18. Thannickal TC1, Moore RY, Nienhuis R, Ramanathan L, Gulyani S, Aldrich M, Cornford M, Siegel JM. Reduced number of hypocretin neurons in human narcolepsy: Neuron. 2000 Sep;27(3):469-474.

19. Karbasforoushan H1, Woodward ND. Resting-state networks in schizophrenia. Curr Top Med Chem. 2012 Dec;12:24042414.

20. Dotti CG, Sullivan CA, Banker GA. The establishment of polarity by hippocampal neurons in culture. J Neurosci. 1988 Apr;8(4):1454-68.

21. da Silva JS, Dotti CG. Breaking the neuronal sphere: regulation of the actin cytoskeleton in neuritogenesis. Nat Rev Neurosci. 2002 Sep;3(9):694-704.

22. Eichberg J, Hauser G. The subcellular distribution of polyphosphoinositides in myelinated and unmyelinated rat brain. Biochim Biophys Acta BBA - Lipids Lipid Metab. 1973 Nov 29;326(2):210-23.

23. Hughes CS, Postovit LM, Lajoie GA. Matrigel: a complexprotein mixture required for optimal growth of cell culture. Proteomics. 2010 May;10(9):1886-90.

24. Pozzi D, Ban J, Iseppon F, Torre V. An improved method for growing neurons: comparison with standard protocols. 
J Neurosci Methods. 2017 Mar 15;280:1-10.

25. Lafon-Cazal M, Adjali O, Galeotti N, Poncet J, Jouin $\mathrm{P}$, Homburger $\mathrm{V}$, et al. Proteomic analysis of astrocytic secretion in the mouse: comparison with the cerebrospinal fluid proteome. J Biol Chem. 2003 Jun 27;278(27):2443848.

26. Landis DM, Weinstein LA, Skordeles CJ. Serum influences the differentiation of membrane structure in cultured astrocytes. Glia. 1990;3(3):212-21.

27. Brewer GJ, Torricelli JR, Evege EK, Price PJ. Optimized survival of hippocampal neurons in B27-supplemented Neurobasal, a new serum-free medium combination. J Neurosci Res. 1993 Aug 1;35(5):567-76.

28. Ivenshitz M, Segal M. Neuronal density determines network connectivity and spontaneous activity in cultured hippocampus. J Neurophysiol. 2010 Aug;104(2):1052-60.

29. Ebrahimi M, Yamamoto $Y$, Sharifi K, Kida H, Kagawa $Y$, Yasumoto $\mathrm{Y}$, et al. Astrocyte-expressed FABP7 regulates dendritic morphology and excitatory synaptic function of cortical neurons. Glia. 2016 Jan;64(1):48-62.

30. Todd GK, Boosalis CA, Burzycki AA, Steinman MQ, Hester LD, Shuster PW, et al. Towards neuronal organoids: a method for long-term culturing of high-density hippocampal neurons. PloS One. 2013;8(4):e58996.

31. Weir K, Blanquie O, Kilb W, Luhmann HJ, Sinning A Comparison of spike parameters from optically identified GABAergic and glutamatergic neurons in sparse cortical cultures. Front Cell Neurosci. 2014;8:460.

32. Tamamaki N, Yanagawa Y, Tomioka R, Miyazaki J-I, Obata K, Kaneko T. Green fluorescent protein expression and colocalization with calretinin, parvalbumin, and somatostatin in the GAD67-GFP knock-in mouse. J Comp Neurol. 2003 Dec 1;467(1):60-79.

33. Murphy TH, Blatter LA, Wier WG, Baraban JM. Spontaneous synchronous synaptic calcium transients in cultured cortical neurons. J Neurosci. 1992 Dec;12(12):4834-45.

34. Cohen E, Ivenshitz M, Amor-Baroukh V, Greenberger V, Segal M. Determinants of spontaneous activity in networks of cultured hippocampus. Brain Res. 2008 Oct 15;1235:2130.

35. Björklund U, Persson M, Rönnbäck L, Hansson E. Primary cultures from cerebral cortex and hippocampus enriched in glutamatergic and GABAergic neurons. Neurochem Res. 2010 Nov;35(11):1733-42.

36. Biffi E, Regalia G, Menegon A, Ferrigno G, Pedrocchi A. The influence of neuronal density and maturation on network activity of hippocampal cell cultures: a methodological study. PLoS ONE. 2013 Dec 27;8(12):e83899.

37. Golshani P, Gonçalves JT, Khoshkhoo S, Mostany R, Smirnakis S, Portera-Cailliau C. Internally mediated developmental desynchronization of neocortical network activity. J Neurosci. 2009 Sep 2;29(35):10890-9.

38. Belle AM, Enright HA, Sales AP, Kulp K, Osburn J, Kuhn $E A$, et al. Evaluation of in vitro neuronal platforms as surrogates for in vivo whole brain systems. Sci Rep. 2018 Jul 17;8:10820.

39. Moskalyuk A, Kooy RF, Giugliano M. Single-cell and neuronal network alterations in an in vitro model of Fragile $X$ syndrome. bioRxiv. 2018 Jul 10;366997.

40. Gonçalves JT, Anstey JE, Golshani P, Portera-Cailliau C. Circuit level defects in the developing neocortex of Fragile $X$ mice. Nat Neurosci. 2013 Jul;16(7):903-9.

41. Testa-Silva G, Loebel A, Giugliano M, de Kock CPJ, Mansvelder HD, Meredith RM. Hyperconnectivity and slow synapses during early development of medial prefrontal cortex in a mouse model for mental retardation and autism. Cereb Cortex N Y N 1991. 2012 Jun;22(6):1333-42.

42. Prè D, Nestor MW, Sproul AA, Jacob S, Koppensteiner $\mathrm{P}$, Chinchalongporn $\mathrm{V}$, et al. A time course analysis of the electrophysiological properties of neurons differentiated from human induced pluripotent stem cells (iPSCs). PloS One. 2014;9(7):e103418.

43. Zhou R, Jiang G, Tian X, Wang X. Progress in the molecular mechanisms of genetic epilepsies using patient-induced pluripotent stem cells. Epilepsia Open. $2018 \mathrm{Jul}$ 8;3(3):331-9.

44. Marton RM, Miura Y, Sloan SA, Li Q, Revah O, Levy RJ, et al. Differentiation and maturation of oligodendrocytes in human three-dimensional neural cultures. Nat Neurosci. 2019 Mar;22(3):484-91.

45. Irons HR, Cullen DK, Shapiro NP, Lambert NA, Lee RH, Laplaca MC. Three-dimensional neural constructs: a novel platform for neurophysiological investigation. J Neural Eng. 2008 Sep;5(3):333-41.

46. Engler AJ, Sen S, Sweeney HL, Discher DE. Matrix Elasticity Directs Stem Cell Lineage Specification. Cell. 2006 Aug 25;126(4):677-89.

47. Lovat V, Pantarotto D, Lagostena L, Cacciari B, Grandolfo M, Righi $\mathrm{M}$, et al. Carbon nanotube substrates boost neuronal electrical signaling. Nano Lett. 2005 Jun;5(6):1107-10.

48. Cellot G, Toma FM, Varley ZK, Laishram J, Villari A, Quintana $M$, et al. Carbon nanotube scaffolds tune synaptic strength in cultured neural circuits: novel frontiers in nanomaterial-tissue interactions. J Neurosci. 2011 Sep 7;31(36):12945-53.

49. Li N, Zhang Q, Gao S, Song Q, Huang R, Wang L, et al. Three-dimensional graphene foam as a biocompatible and conductive scaffold for neural stem cells. Sci Rep. 2013;3:1604.

50. Posypanova GA, Gayduchenko IA, Moskaleva EY, Fedorov GE. Neuronal differentiation of PC12 cells and mouse neural stem cells on carbon nanotube films. Cell Tissue Biol. 2016 May 1;10(3):194-201.

51. Ulloa Severino FP, Ban J, Song Q, Tang M, Bianconi G, Cheng $G$, et al. The role of dimensionality in neuronal network dynamics. Sci Rep. 2016 Jul 11;6:29640.

52. Pampaloni NP, Lottner M, Giugliano M, Matruglio A, D'Amico $\mathrm{F}$, Prato $\mathrm{M}$, et al. Single-layer graphene modulates neuronal communication and augments membrane ion currents. Nat Nanotechnol. 2018;13(8):755-64

53. Bosi S, Rauti R, Laishram J, Turco A, Lonardoni D, Nieus $\mathrm{T}$, et al. From 2D to 3D: novel nanostructured scaffolds to investigate signalling in reconstructed neuronal networks. Sci Rep. 2015;5:9562

54. Aurand ER, Usmani S, Medelin M, Scaini D, Bosi S, Rosselli FB, et al. Nanostructures to engineer 3D neural-interfaces: directing axonal navigation toward successful bridging of spinal segments. Adv Funct Mater. 28(12):1700550.

55. Musick KM, Rigosa J, Narasimhan S, Wurth S, Capogrosso $\mathrm{M}$, Chew DJ, et al. Chronic multichannel neural recordings from soft regenerative microchannel electrodes during gait. Sci Rep. 2015 Sep 24;5:14363.

56. Park D-W, Ness JP, Brodnick SK, Esquibel C, Novello J, Atry $\mathrm{F}$, et al. Electrical neural stimulation and simultaneous in vivo monitoring with transparent graphene electrode arrays implanted in GCaMP6f mice. ACS Nano. 2018 23;12(1):148-57.

57. Masvidal-Codina E, Illa X, Dasilva M, Calia AB, Dragojević T, Vidal-Rosas EE, et al. High-resolution mapping of infraslow cortical brain activity enabled by graphene microtransistors. Nat Mater. 2019 Mar;18(3):280-8.

58. Li S, Severino FPU, Ban J, Wang L, Pinato G, Torre V, et al. Improved neuron culture using scaffolds made of three-dimensional PDMS micro-lattices. Biomed Mater. 2018;13(3):034105.

59. Wei J, Shi J, Wang B, Tang Y, Tu X, Roy E, et al. Fabrication of adjacent micropillar arrays with different heights for cell studies. Microelectron Eng. 2016 Jun;158:22-5.

60. Nagayama K, Inoue T, Hamada Y, Matsumoto T. A novel patterned magnetic micropillar array substrate for analysis of cellular mechanical responses. J Biomech. 2017 Dec 8;65:194-202

61. Migliorini E, Ban J, Grenci G, Andolfi L, Pozzato A, Tormen M, 
et al. Nanomechanics controls neuronal precursors adhesion and differentiation. Biotechnol Bioeng. 2013;110(8):2301-10.

62. Wei J, Pozzi D, Ulloa Severino FP, Torre V, Chen Y. Fabrication of PLGA nanofibers on PDMS micropillars for neuron culture studies. Microelectron Eng. 2017 May;175(C):67-72.

63. D'Aiuto L, Naciri J, Radio N, Tekur S, Clayton D, Apodaca $G$, et al. GABAergic hub neurons orchestrate synchrony in developing hippocampal networks. Science. 2009 Dec 4;326(5958):1419-24.

65. Sanchez-Vives MV, McCormick DA. Cellular and network mechanisms of rhythmic recurrent activity in neocortex. Nat Neurosci. 2000 Oct;3(10):1027-34.

66. Compte A, Reig R, Descalzo VF, Harvey MA, Puccini GD, Sanchez-Vives MV. Spontaneous high-frequency $(10-80 \mathrm{~Hz})$ oscillations during up states in the cerebral cortex in vitro. $J$ Neurosci. 2008 Dec 17;28(51):13828-44.

67. Gähwiler BH, Capogna M, Debanne D, McKinney RA, Thompson SM. Organotypic slice cultures: a technique has come of age. Trends Neurosci. 1997 Oct;20(10):471-7.

68. Stoppini L, Buchs PA, Muller D. A simple method for organotypic cultures of nervous tissue. J Neurosci Methods. 1991 Apr;37(2):173-82.

69. Magalhães DM, Pereira N, Rombo DM, Beltrão-Cavacas C, Sebastião AM, Valente CA. Ex vivo model of epilepsy in organotypic slices-a new tool for drug screening. J Neuroinflammation. 2018 Jul 11;15(1):203.

70. Allene C, Picardo MA, Becq H, Miyoshi G, Fishell G, Cossart R. Dynamic changes in Interneuron morphophysiological properties mark the maturation of hippocampal network activity. J Neurosci. 2012 May 9;32(19):6688-98.

71. Crépel V, Aronov D, Jorquera I, Represa A, Ben-Ari Y, Cossart R. A parturition-associated nonsynaptic coherent activity pattern in the developing hippocampus. Neuron. 2007 Apr 5;54(1):105-20.

72. Okamoto K, Ishikawa T, Abe R, Ishikawa D, Kobayashi C, Mizunuma $\mathrm{M}$, et al. Ex vivo cultured neuronal networks emit in vivo-like spontaneous activity. J Physiol Sci. 2014 Nov;64(6):421-31.

73. Cobb SR, Buhl EH, Halasy K, Paulsen O, Somogyi P. Synchronization of neuronal activity in hippocampus by individual GABAergic interneurons. Nature. 1995 Nov 2;378(6552):75-8.

74. Galarreta M, Hestrin S. A network of fast-spiking cells in the neocortex connected by electrical synapses. Nature. 1999 Nov 4;402(6757):72-5.

75. Gentet LJ, Avermann M, Matyas F, Staiger JF, Petersen $\mathrm{CCH}$. Membrane potential dynamics of GABAergic neurons in the barrel cortex of behaving mice. Neuron. 2010 Feb;65(3):422-35.

76. Wickham J, Brödjegård NG, Vighagen R, Pinborg LH, Bengzon J, Woldbye DPD, et al. Prolonged life of human acute hippocampal slices from temporal lobe epilepsy surgery. Sci Rep. 2018 Mar 7;8(1):1-13.

77. Qi X-R, Verwer RWH, Bao A-M, Balesar RA, Luchetti S, Zhou J-N, et al. Human brain slice culture: a useful tool to study brain disorders and potential therapeutic compounds. Neurosci Bull. 2019 Apr;35(2):244-52. 